\title{
Les nouveaux outils de la régulation médicale en Samu-Centre 15
}

\author{
New Tools for Medical Regulation in an Emergency Medical Communication Center
}

\author{
F. Braun \\ (C) SFMU et Lavoisier SAS 2017
}

La régulation médicale est un acte médical pratiqué au téléphone par un médecin régulateur d'un centre d'appels, en réponse à une demande concernant un patient se trouvant à distance en situation d'urgence [1].

La télémédecine concerne «les actes médicaux, réalisés à distance, au moyen d'un dispositif utilisant les technologies de l'information et de la communication » [2]. La réponse médicale apportée dans le cadre de la régulation médicale est un acte de télémédecine. La télémédecine est une des formes de coopération dans l'exercice médical, mettant en rapport à distance, grâce aux technologies de l'information et de la communication, un patient (et/ou les données médicales nécessaires) et un ou plusieurs médecins et professionnels de santé, à des fins médicales de diagnostic, de décision, de prise en charge et de traitement dans le respect des règles de la déontologie médicale [3].

Dans le cadre de son exercice, basé sur des recommandations de la Haute Autorité de Santé (HAS) [4], et pour pratiquer le « juste soin », le médecin régulateur doit colliger le maximum d'informations sur son patient et les circonstances de son appel [5]. La recherche de ces informations est une phase essentielle de la régulation médicale qui, pourtant, ne doit pas être chronophage et retarder la décision médicale. Cette information doit avoir différentes origines :

- dossier médical du patient ;

- données «paracliniques » relevées par le patient lui-même ou par un intervenant ;

- données « cliniques » relayées par la visioconférence ou tout moyen permettant de voir le patient.

\section{Le dossier médical}

L'accès au dossier médical personnel par le médecin régulateur du Samu apparaît comme une solution intéressante dès

\section{F. Braun $(\bowtie)$}

Président de Samu-Urgences de France, 103 boulevard Magenta, 75010 Paris, France

e-mail : francois.braun@sudf.fr lors qu'il répond à des contraintes temporelles liées à l'exercice même de la médecine d'urgence [6] : une minute pour le médecin régulateur ayant à déterminer une réponse immédiate à un appel, quinze minutes pour les autres situations d'urgence. Deux solutions peuvent être envisagées pour accéder rapidement à une information pertinente :

- réaliser une synthèse du dossier, immédiatement accessible : c'est le choix effectué pour les patients des Établissements d'Hébergement pour Personnes Âgées Dépendantes (EHPAD) dans le cadre du dossier de liaison d'urgence (DLU) [7] défini par la HAS ;

- bénéficier de l'aide d'un outil informatique permettant d'aller directement à l'essentiel dans le DMP, sans qu'aucune synthèse n'en ait été préalablement réalisée [5]: c'est la solution proposée par Samu-Urgences de France au GIP-DMP qui a donné naissance au projet de LEcture Rapide en Urgence du Dossier Informatisé patient (LERUDI) [8,9].

\section{Les données « paracliniques »}

La télétransmission de données concernant le patient pris en charge débute en 1966 au Samu de Toulouse avec la transmission de l'électrocardiographe et de la fréquence respiratoire. Le matériel, encombrant et complexe, sera abandonné mais l'idée même de télémédecine était née [10]. Les recommandations sur la prise en charge de l'infarctus du myocarde et l'importance de «l'ECG qualifiant » ont vu renaître l'attrait de la télémédecine et de la télétransmission des ECG dès le début des années 1990. La pertinence diagnostique des urgentistes ayant alors rapidement été démontrée, cet " usage » a été limité à des médecins éloignés et isolés. Dès le début des années 2000, la société européenne de télémédecine s'est rapidement intéressée à l'utilisation de cet outil dans le cadre de l'urgence. Rapidement les Samu ultramarins se saisissaient de cette opportunité pour améliorer la prise en charge des patients isolés [11-13]. Plus récemment, l'intérêt de la télétransmission de l'ECG au médecin 
régulateur, sur sa demande, par des secouristes professionnels a été étudié et semble démontré [14].

Le développement de nouveaux réseaux de télémédecine, principalement dans le cadre du suivi de pathologies chroniques comme l'insuffisance cardiaque (ICALOR ${ }^{\circledR}$, OSI$\mathrm{CAT}^{\circledR} \ldots$ ), génère des flux d'informations médicales dont les Samu-Centre 15 sont malheureusement privés et qui seraient certainement utiles aux médecins régulateurs sollicités lors des décompensations aiguës de ces pathologies. L'idée d'un lien systématique de ces réseaux avec la régulation médicale doit être entendue, comprise et défendue par nos autorités de tutelle.

\section{Les données cliniques}

Par opposition aux données paracliniques, issues d'appareils de mesure, les données cliniques concernent plus directement l'utilisation des « sens » du médecin régulateur. $\mathrm{Si}$ l'ouïe est le premier sens utilisé en régulation médicale, et encore le seul, la vue peut maintenant participer à la décision médicale. L'utilisation de lunettes connectées montre désormais sa facilité et sa fiabilité [15] lors d'un bilan passé au médecin régulateur par des ambulanciers ou des personnels paramédicaux mais aussi pour aider le médecin sur les lieux lors de la réalisation d'actes complexes nécessitant parfois le recours à un médecin spécialisé (gynéco-obstétricien lors d'un accouchement difficile par exemple).

La diffusion massive des smartphones doit nous interpeller sur la possibilité pour un appelant non seulement de renseigner la régulation médicale sur sa localisation via les coordonnées GPS ${ }^{\circledR}$ de son appareil, mais également de rendre visible la « scène » au médecin régulateur via les possibilités de son appareil. Utilisée à l'étranger, comme par exemple Megan David Adom ${ }^{\circledR}$ en Israël, cette technologie sera rapidement disponible en France et révolutionnera l'acte de régulation médicale.

Par ces exemples nous tenons à montrer le dynamisme des urgentistes et des assistants de régulation médicale œuvrant au sein des plateformes de régulation médicale « Samu-Santé », dynamisme qui pousse à l'intégration des différents flux d'information et de communication dans l'acte de régulation médicale. Gageons que l'histoire ne s'arrête pas là et que, demain, les évolutions technologiques permettent d'améliorer encore la prise en charge des patients faisant appel à ce remarquable outil créé il y a plus de 50 ans par des pionniers visionnaires.

Liens d'intérêts : L'auteur déclare ne pas avoir de lien d'intérêt.

\section{Références}

1. Giroud M (2009) La régulation médicale en médecine d'urgence. Réanimation 18:737-41

2. République Française (2010) Décret $n^{\circ} 2010-1229$ du 19 octobre 2010 relatif à la télémédecine. https://www.legifrance.gouv.fr/ affichTexte.do?cidTexte=JORFTEXT000022932449\&categorieLien=id (Dernier accès le 11 septembre 2017)

3. Télémédecine. Les préconisations du Conseil Nationale de l'Ordre des Médecins. Janvier 2009 https://www.conseil-national. medecin.fr/sites/default/files/telemedecine2009.pdf (Dernier accès le 11 septembre 2017)

4. Giroud M, Pateron D (2012) Régulation médicale : la HAS édicte des recommandations sur la prise en charge d'un appel. Ann Fr Med Urgence 2:4-6

5. Braun F, Berthier F (2009) Les interconnexions de la régulation médicale. Congrès Urgences 2009 (Paris) 83:817-29

6. Giroud M (2009) L'accès au dossier médical personnel par le médecin régulateur du Samu. Congrès Urgences 2009 (Paris) 80:807-16

7. https://www.has-sante.fr/portail/jcms/c_2049090/fr/dossier-deliaison-d-urgence-dlu (Dernier accès le 24 juillet 2017)

8. Lobel E, Janin N, Janeczek AL, et al (2012) LERUDI : lecture rapide en urgence du dossier informatisé patient. Congrès Urgences 2012 (Paris)

9. Gayet P, Charlet J, Janin N, et al (2017) Une synthèse du dossier médical pour décider aux urgences : le projet LERUDI. Ann Fr Med Urgence 7:166-73

10. Cazalaa JB (2015) Anesthésie, analgésie, réanimation, Samu : notre histoire de 1945 aux années 2000. Tome II : Le Samu. Éditions Glyphe, Paris

11. Buchs A (2004) Télémédecine et médecine d'urgence en Polynésie française : état des lieux, enjeux et perspectives de développement. Rev Samu 168:39-41

12. Le Guen T (2005) La télémédecine en Guyane. Rev Samu 174:713

13. Runavot G (2005) Télémédecine et site isolé : projet dans le site de Mafate à l'Île de la Réunion. Rev Samu 174:15-8

14. Frattini B, Diegelmann P, Klein I, et al (2015) Étude de faisabilité de la réalisation et de la télétransmission d'électrocardiogramme par des équipes de premier secours. http://www.sfmu.org/upload/ 70_formation/02_eformation/02_congres/Urgences/urgences2015/donnees/fs-recherche/fs_resume_415.htm

15. Schmutz T, Braun F (2016) Lunettes connectées : médecins régulateurs, ouvrez les yeux! Ann Fr Med Urgence 6:339 